Wright State University

CORE Scholar

Kno.e.sis Publications

The Ohio Center of Excellence in Knowledge-

Enabled Computing (Kno.e.sis)

2015

\title{
Knowledge-Driven Personalized Contextual mHealth Service for Asthma Management in Children
}

\author{
Pramod Anantharam \\ Wright State University - Main Campus, anantharam.2@wright.edu \\ Tanvi Banerjee \\ Wright State University - Main Campus, tanvi.banerjee@wright.edu \\ Amit P. Sheth \\ Wright State University - Main Campus, amit@sc.edu \\ Krishnaprasad Thirunarayan \\ Wright State University - Main Campus, t.k.prasad@wright.edu \\ Surendra Marupudi \\ Wright State University - Main Campus, marupudi.3@wright.edu
}

See next page for additional authors

Follow this and additional works at: https://corescholar.libraries.wright.edu/knoesis

Part of the Bioinformatics Commons, Communication Technology and New Media Commons, Databases and Information Systems Commons, OS and Networks Commons, and the Science and

Technology Studies Commons

\section{Repository Citation}

Anantharam, P., Banerjee, T., Sheth, A. P., Thirunarayan, K., Marupudi, S., \& Sridharan, V. (2015). KnowledgeDriven Personalized Contextual mHealth Service for Asthma Management in Children. .

https://corescholar.libraries.wright.edu/knoesis/1068

This Conference Proceeding is brought to you for free and open access by the The Ohio Center of Excellence in

Knowledge-Enabled Computing (Kno.e.sis) at CORE Scholar. It has been accepted for inclusion in Kno.e.sis Publications by an authorized administrator of CORE Scholar. For more information, please contact library-

corescholar@wright.edu. 


\section{Authors}

Pramod Anantharam, Tanvi Banerjee, Amit P. Sheth, Krishnaprasad Thirunarayan, Surendra Marupudi, and Vaikunth Sridharan

This conference proceeding is available at CORE Scholar: https://corescholar.libraries.wright.edu/knoesis/1068 


\title{
Knowledge-driven Personalized Contextual mHealth Service for Asthma Management in Children
}

\author{
Pramod Anantharam, Tanvi Banerjee, Amit Sheth, Krishnaprasad Thirunarayan, Surendra Marupudi, Vaikunth Sridharan \\ Ohio Center of Excellence in Knowledge-enabled Computing (Kno.e.sis), \\ Wright State University \\ Dayton, $\mathrm{OH}$ \\ \{pramod@knoesis.org, tanvi@knoesis.org, amit@knoesis.org, tkprasad@knoesis.org, surendra@knoesis.org, vaikunth@knoesis.org\} \\ Shalini G. Forbis, MD, MPH \\ Dept of Pediatrics, \\ Wright State University Boonshoft School of Medicine, Dayton, OH \\ \{ForbisS@childrensdayton.org\}
}

\begin{abstract}
Wide adoption of smartphones and availability of low-cost sensors has resulted in seamless and continuous monitoring of physiology, environment, and public health notifications. However, personalized digital health and patient empowerment can become a reality only if the complex multisensory and multimodal data is processed within the patient context. Contextual processing of patient data along with personalized medical knowledge can lead to actionable information for better and timely decisions. We present a system called kHealth capable of aggregating multisensory and multimodal data from sensors (passive sensing) and answers to questionnaire (active sensing) from patients with asthma. We present our preliminary data analysis comprising data collected from real patients highlighting the challenges in deploying such an application. The results show strong promise to derive actionable information using a combination of physiological indicators from active and passive sensors that can help doctors determine more precisely the cause, severity, and control level of asthma. Information synthesized from kHealth can be used to alert patients and caregivers for seeking timely clinical assistance to better manage asthma and improve their quality of life.
\end{abstract}

\section{INTRODUCTION}

Asthma affects over 300 million people claiming over 250,000 lives worldwide annually. Asthma management is challenging; as a multifactorial disease with subjective causes and symptoms, extant diagnosis and treatment guidelines can be improved with evidence-based action recommendation. More than 25 million people in the U.S. are diagnosed with asthma, out of which 7 million are children [1]. Asthma related healthcare costs alone are around $\$ 50$ billion a year [2]. Current reactive healthcare costs more than 17\% of GDP in the US alone [3], [4]. Specifically, there were 155,000 hospital admissions and 593,000 ER visits in 2006 [5]. It is estimated that, by 2025 , over 400 million people will be affected by asthma worldwide. With increasing adoption of mobile devices and low-cost sensors, an unprecedented amount of data is being collected by people [6]. However, this huge amount of data needs to be converted into actionable information that can a) help detect asthma triggers for an individual and b) provide relevant information to the clinician that can help treat the chronic illness.
In this study, we describe our preliminary work in deriving actionable information from data collected from children diagnosed with asthma by adapting our kHealth kit [7], a smart mobile application with sensors, to capture timely information about triggers and symptoms. We discuss our preliminary data collection and feature extraction results from four subjects (children with asthma symptoms from ages 5 to 17) using our kHealth kit that has been found very informative by our clinical collaborator. This application can provide crucial insights into the environmental conditions of an asthma patient as well as the triggers that can affect an individual asthma patient. These insights can help clinicians diagnose, monitor progression, and treat the illness and improve health management for the patients.

There are three broad categories of mHealth or digital health applications. Telemedicine [8] provides techniques to exchange medical information through electronic communication. Cloud based healthcare solutions are gaining momentum where all the healthcare information is pushed to a cloud. However, they remain vulnerable to hacking and other forms of unauthorized dissemination of data through security breach. Intelligence at the edge [9] takes an orthogonal approach to cloud and telemedicine based approaches by using mobile devices local to patients for computation where data remain fully under patient control and computation is performed on the mobile device. Intelligence at the edge aspires to provide proactive, personalized, and actionable information to patients and doctors for evidence-based decision support. kHealth kit for asthma utilizes the principles of Intelligence at the edge and the rationale with this choice is explained in the next section.

\section{Motivation AND Related Work ON Asthma MANAGEMENT}

The availability of low-cost $(<\$ 100)$ sensors and mobile devices for monitoring physiological, physical, environmental, and cognitive health within human bodies [10], [11], on humans [12], [13], [14] and around humans [15], [16], [17], [18], [19] is revolutionizing healthcare [20]. Microsoft Kinect and on-board sensors on mobile phones are being increasingly adopted in assisted living environments and hospitals for monitoring daily activities [21], [22], [23], [24], [25] and to aid in 
the mitigation of falls [26], [27]. Ingestible sensors are radicalizing diagnosis and understanding of disease progression. The FDA has approved the first ingestible sensor by Proteus Digital Health for monitoring compliance with medication [28]. Mobile communication and sensing corporations are incentivizing relevant research via competitions and rewards such as the Qualcomm Tricorder X Prize (\$10 million) [29] and the Nokia Sensing X Challenge (\$2.5 million) [30]. This is promoting the development of innovative sensing and data interpretation techniques. Many research groups such as the Center for Body Computing [31], Wyss Institute at Harvard [14], and the Harvard Sensor Networks Lab (e.g., CodeBlue project) [32], and the community funding sources such as Kickstarter [33] and the e-Health platform [34] have propelled innovations in integrating low-cost sensors with mobile devices.

Patient involvement and active partnership are necessary in the prevention and proactive control of chronic ailments. Asthma symptom detection using sensors for monitoring wheezing sound patterns [35], mechanical vibrations, respiration (using radio waves), Nitric Oxide in breath, etc., have been explored [36], [37], [38]. iSonea [39], in collaboration with Qualcomms 2net cloud-based platform effort [40], [29], uses acoustic signals for detection and sharing of asthma information. However, variability in wheezing patterns across patients makes it a weak indicator [41], [42]. A wireless body sensor network can aggregate other asthma related data using a GPS, accelerometer, and particulate matter monitor [43]. Beyond machine sensors, personal observations obtained from patients through questionnaires can provide complementary information [44]. A sensor from Propeller Health (formerly, Asthmapolis) records inhaler use and its geographical location to assess asthma attacks in the community [45], and caregivers and patients can register to obtain alerts. Compliance to medication by using an alerting mechanism is studied by [46]. The Manchester Asthma and Allergy Study, started in 1995 at Wythenshawe Hospital (UHSM), Manchester, in partnership with Microsoft Research Cambridge [47], is an active birth cohort study with promising outcomes [48] that aims at understanding the role of genetics and the influence of environmental factors on asthma.

Our research focus is on understanding: (1) personal and public health signals influencing asthma beyond the NHLBI asthma guidelines [49], (2) ranking these based on their influence on asthma, and (3) ultimately providing actionable information to patients and doctors. The public health information and studies provide meaningful baseline and priors. Personalization is crucial since the triggers and treatment plans are subjective. In contrast with the existing mobile applications, which are journaling in nature in that they keep track of symptoms and send medication reminders via SMS or other means, the proposed evidence-based approach will build on them by enabling timely determination of asthma triggers, overcoming underreporting problem and facilitating more precise diagnosis and action recommendation.

\section{ASthma: Symptoms, Control AND TREatment}

The severity of asthma can be categorized as shown in Table I, and corresponding control levels and recommended action are shown in Table II. Children with asthma require ongoing care and continual reassessment. The treatment of

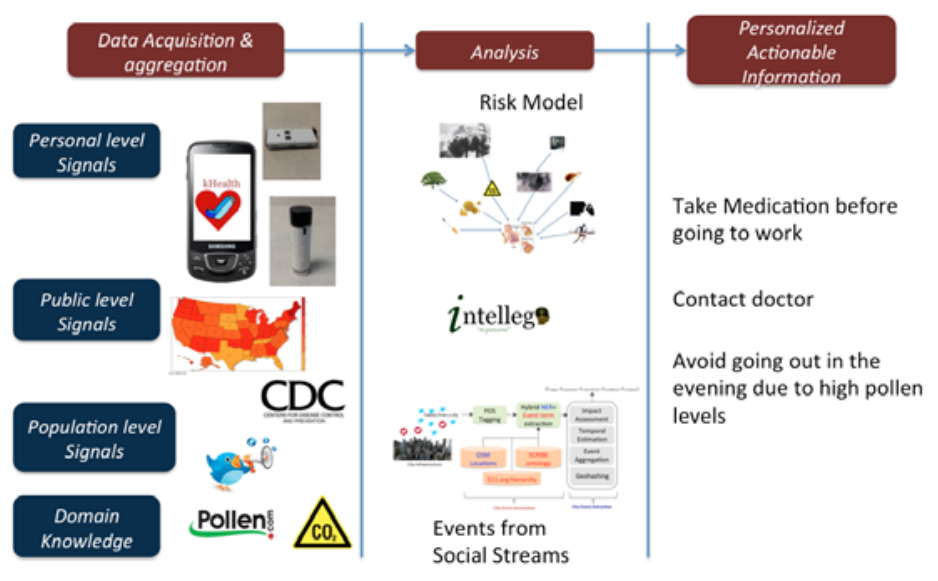

Fig. 1. kHealth data ingestion and processing pipeline demonstrating data aggregation, analysis, and actionable information

asthma is complicated by the fact that impairment (daytime and nighttime symptoms, activity limitation) can vary during the year (e.g., with seasons, or specific allergens) and also change over the life of the child, e.g., some children may see asthma severity change over time during adolescence. These factors necessitate the ongoing monitoring of children, including the assessment of disease control, adjustment of medications, consideration of factors that may worsen asthma and ongoing patient/family education [49].

Asthma control is assessed as being well controlled, not well controlled or poorly controlled. Due to the seasonal variation in asthma symptomatology for some patients, medications is not decreased until these patients have maintained wellcontrolled asthma for one year. Well-controlled patients (if 3 months) can be seen every six months [49].

"Not well controlled" asthma patients have symptoms more than two days per week (or multiple times on two or less days/week) and may need to increase their level of medications, e.g., a patient on a low dose of inhaled corticosteroid may need to be placed on a medium dose. These patients will need to be seen more often until their asthma has been well controlled for at least three months. Other considerations in determining frequency of follow-up visits for asthma may include the need for ongoing education, assessment of inhaler technique, or for maintaining preventive therapies.

Patients with poorly controlled asthma have symptoms such as cough or wheeze throughout the day, and this will manifest itself as a severe activity limitation. These children will typically need to utilize a SABA inhaler multiple times/day. The patients that have three or more asthma exacerbations in a year require oral steroids for treatment and may need to be referred to a specialist.

\section{KHEALTH}

kHealth is a knowledge-enabled semantic platform to enhance decision making and improve health, fitness, and wellbeing. It supports contextual (e.g., condition specific) and personalized (e.g., patient specific) annotation, integration, and interpretation of sensor and mobile data from using deep domain (e.g., disease) specific knowledge. kHealth is currently being investigated for applications in (a) reducing readmission 
TABLE I. ASTHMA SEVERITY LEVELS AND CORRESPONDING INTENSITY OF SYMPTOMS

\begin{tabular}{|l|l|l|l|}
\hline Intermittent Asthma & Mild Persistent Asthma & Moderate Persistent Asthma & Severe Persistent Asthma \\
\hline Symptoms $<2$ days/week & Symptoms at least 2 days/week & Symptoms daily & Symptoms daily and all the time \\
\hline No night time awakening & $\begin{array}{l}\text { Night time awakening } \leq 2 \text { times / } \\
\text { month }\end{array}$ & $\begin{array}{l}\text { Night time awakening 4 times / } \\
\text { month }\end{array}$ & $\begin{array}{l}\text { Night time awakening }>4 \\
\text { times/month }\end{array}$ \\
\hline $\begin{array}{l}\text { Zero/One exacerbation with the use } \\
\text { of corticosteroid }\end{array}$ & $\begin{array}{l}\text { Exacerbation requiring oral corti- } \\
\text { costeroid 2-4 per year }\end{array}$ & $\begin{array}{l}\text { Exacerbation requiring oral corti- } \\
\text { costeroid 2-4 per year }\end{array}$ & $\begin{array}{l}\text { Exacerbation requiring oral corti- } \\
\text { costeroid 2-4 per year }\end{array}$ \\
\hline Normal lung function & $\begin{array}{l}\text { Lung function }>80 \% \text { of predicted } \\
\text { FEV1 }\end{array}$ & $\begin{array}{l}\text { Lung function 60 - 80\% predicted } \\
\text { Lung function }<60 \% \text { predicted }\end{array}$ \\
\hline
\end{tabular}

TABLE II. ASTHMA CONTROL LEVELS AND RECOMMENDED ACTIONS DEPENDING ON THE ASTHMA SEVERITY LEVEL

\begin{tabular}{|l|l|l|l|}
\hline Asthma Control => & $\begin{array}{l}\text { Daily Medication Choices for } \\
\text { starting therapy }\end{array}$ & Not Well Controlled & Poor Controlled \\
\hline Severity Level of Asthma & (Recommended Action) & (Recommended Action) & (Recommended Action) \\
\hline Intermittent Asthma & SABA prn & - & - \\
\hline Mild Persistent Asthma & Low dose ICS & Medium ICS & Medium ICS \\
\hline Moderate Persistent Asthma & $\begin{array}{l}\text { Medium dose ICS alone Or with } \\
\text { LABA/montelukast }\end{array}$ & $\begin{array}{l}\text { Medium ICS + LABA/Montelukast } \\
\text { Or High dose ICS }\end{array}$ & $\begin{array}{l}\text { Medium ICS + LABA/Montelukast } \\
\text { Or High dose ICS* }\end{array}$ \\
\hline Severe Persistent Asthma & $\begin{array}{l}\text { High dose ICS with } \\
\text { LABA/montelukast }\end{array}$ & Needs specialist care & Needs specialist care \\
\hline
\end{tabular}

of discharged Acute Decompensated Heart Failure (ADHF) patients, (b) management of Asthma in children, for which Institutional Review Board (IRB) approvals have been obtained and early testing of kHealth kit with patients have been completed as discussed here, (c) GI readmission, for which an application has been developed, and (d) geriatrics for which research and development work continues [50]. All applications involve active clinical collaborations with medical professionals leading to evaluation with patients. We will present a preliminary deployment study and summarize the data we have collected from kHealth for Asthma application.

\section{KHEALTH FOR ASTHMA}

Increasing availability of open data and easy accessibility has resulted in a wide variety of observations that may benefit proactive and personalized asthma management. We have consolidated a list of data sources and possible health related information we can glean from the data sources in Table III. There are multiple sources providing valuable information at personal and public levels. Personal level signals are specific to a patient and are crucial for understanding the asthma symptomatic variations of the patient. Public level signals include environmental observations that may impact an asthma patient. The public level signals are acquired by applying location and time constraints to filter out signals for increased relevance to a patient. Population level observations include symptomatic report of asthma incidents.

\section{A. Actionable Information for Asthma}

We demonstrate various facets of asthma management by considering a concrete scenario. Consider Mr. Smith who is suffering from asthma for the last 5 years. His doctor has diagnosed him with mild persistent asthma (Table I) and a control level of "Not well controlled". Mr. Smith and his doctor are concerned about the late night asthma attacks Mr. Smith is having at home since continued asthma attacks may lead to change in severity diagnosis. Mr. Smith needs a recommendation on the possible measures he can take to avoid asthma attacks at home. Figure 2 depicts the scenario of Mr. Smith along with personal, public, and population level observations. Mr. Smith needs an answer to his question "How can I reduce my asthma attacks at night?". This question is broken down into component questions shown in colored boxes. We characterize the data sources into three broad categories (a) personal level, (b) population level, and (c) public level signals. Answers to component questions require an analysis of observations spanning all the three data sources. A holistic consideration is necessary for answering all the component questions and effectively answering the question for Mr. Smith.

Figure 2 exemplifies each data source category with concrete examples. Component questions are placed in the data source category based on the data requirements to answer the component questions. Personal level signals include environmental (such as temperature, humidity, luminosity, and carbon-monoxide) and physiological (such as exhaled nitric oxide, heart rate, and lung capacity) observations. The indoor air quality, the potential triggers, and the wheezing level can be determined by personal level observations using the carbon monoxide sensor, dust sensor, and sound recording respectively. The exposure level over day can be determined utilizing public sources reporting pollen, weather and air quality. The propensity toward asthma can be determined using demographic, location, and prevalence information. We can then synthesize a comprehensive and reliable answer to Mr. Smith's query. In Figure 2, a strong correlation between carbonmonoxide and luminosity is observed. There may be multiple such correlations but understanding that carbon-monoxide or indoor air quality in general has significant influence on asthma attacks is deemed important from domain experts. If this information is provided to Mr. Simth and his doctor, they can mitigate the problem of asthma attacks effectively. Mr. Smith is asked by his doctor to close the windows of the house before he leaves to work and take a route that has the best air quality. Such an action is possible due to the holistic analysis of personal, public, and population level signals.

\section{B. kHealth Kit for Asthma}

There are three major components in the kHealth kit for asthma. We provide some details on each of the components along with some design choices for each of them here:

Sensors: Considering the above scenario, we selected the most promising data modalities to be captured from asthma patients. kHealth kit for asthma shown in Figure 3 consists of 

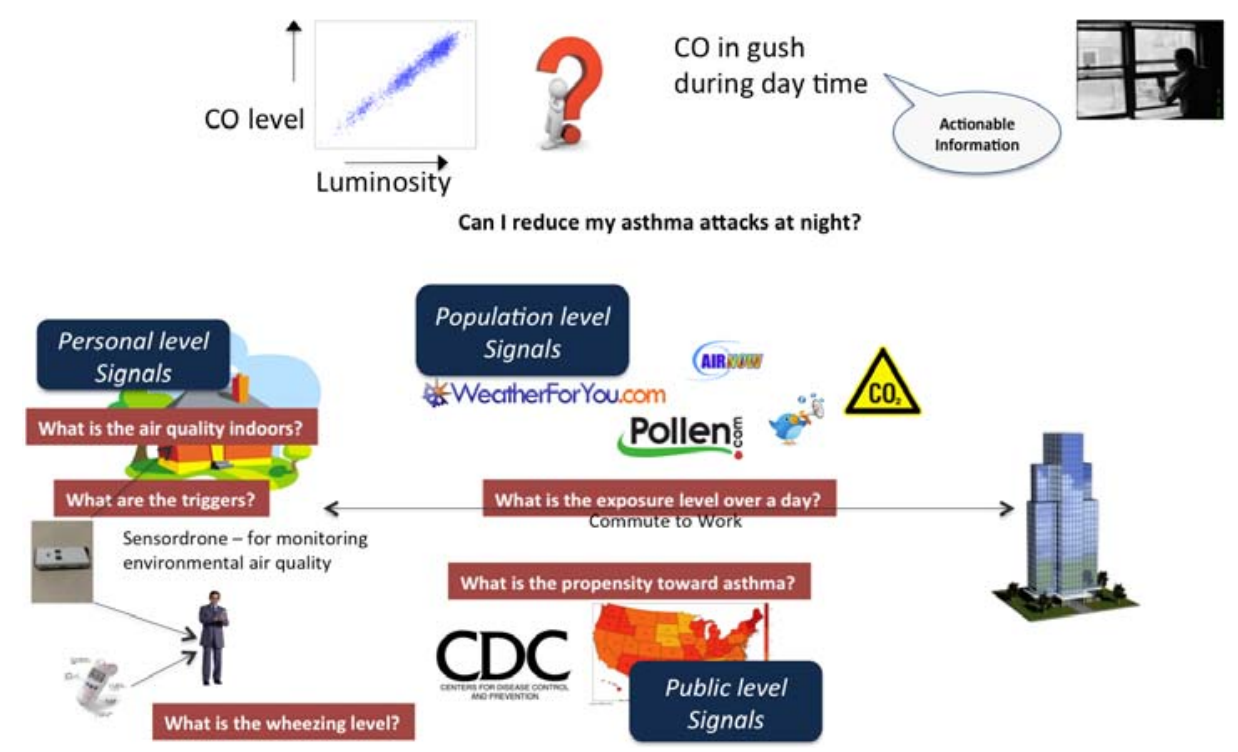

Fig. 2. Asthma management scenario involving personal, public, and population level observations to synthesize actionable information

TABLE III. HEALTH SIGNALS AND THEIR SOURCES THAT WILL BE LEVERAGED BY OUR ALGORITHMS FOR CONTINUOUS, PROACTIVE, AND PREVENTIVE ANALYTICS FOR ASTHMA MANAGEMENT.

\begin{tabular}{|l|l|l|}
\hline & Data Sources & Health Signals \\
\hline Personal Level & $\begin{array}{l}\text { Physiological: Wheezometer, Nitric Oxide, Accelerometer, } \\
\text { Microphone, Contextual Questions; Environmental: Sensor- } \\
\text { drone, Dust Sensor, Location }\end{array}$ & $\begin{array}{l}\text { Wheezing sound, Exhaled Nitric Oxide, Activity level, } \\
\text { Coughing sound Personal observations, Temperature, Hu- } \\
\text { midity, CO } \text {, Luminosity, Proximity, Altitude, Pressure, }_{\text {Dust. Particles, Indoor/Outdoor }}\end{array}$ \\
\hline Public Level & $\begin{array}{l}\text { Everyaware, AirQuality Egg, Allergy Alerts, Social Obser- } \\
\text { vations (e.g., tweets), Air Quality Index, CDC, DCHCs EMR } \\
\text { Records (periodic manual review) }\end{array}$ & $\begin{array}{l}\text { Community shared air pollution information, Air pollutants } \\
\text { outdoors, Pollen level due to weeds, tree, grass, and mold, } \\
\text { Air pollution and asthma symptoms and incidents, Asthma } \\
\text { prevalence based on aggregate demographics and severity }\end{array}$ \\
\hline
\end{tabular}
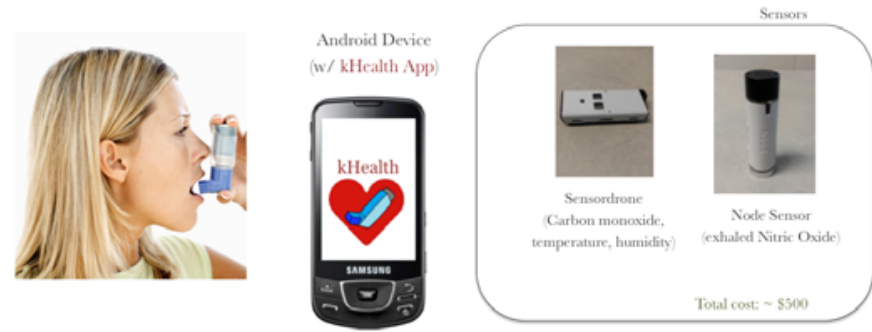

Along with two sensors in the kit, the application uses a variety of population level signals from the web:

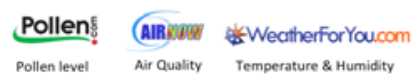

Fig. 3. kHealth kit with personal, population, and public level observations utilized in our study

a mobile device (android phone or tablet), sensordrone and NODE sensor platforms. Sensordrone is primarily used for continuous collection of indoor environmental and air quality observations. NODE sensor monitors the exhaled nitric-oxide which indicates inflammation in lungs; an evidence of reaction to a trigger. We characterize sensing into two broad categories: (a) passive sensing: machine sensors collects observations without involving people in the loop and (b) active sensing: requires involvement of people for collecting these observations e.g., answering a questionnaire. The sampling rate of sensordrone is every second i.e., we collect temperature, humidity, and carbon-monoxide once every second. NODE reading results in one sample per reading.

Mobile Application: An interface for collecting the observations of interest by describing a sequence of steps to the patient or parents of children with asthma is provided by the mobile application. There are two main things the application does: (1) collects all the observations from sensors chosen for our study using Bluetooth and (2) registers answers to questionnaire that may serve as ground truth for later corroboration and utilization of sensor observations. There are multiple features such as reminders to take readings once a day, view historical observations, and view summary of all the answers to questionnaire.

Summary: Sensor observations are summarized using a time series plot. Answers to questionnaire are summarized using a table. The table aggregates summary for the entire data collection period and provides a quick feedback to the patients. The questionnaire is designed such that the answers aggregated over time can be utilized to synthesize the control level. Estimated control level can then be utilized with severity level defined in Table I to recommend actions outlined in Table II. Subject to the limitations of the current IRB protocol, no actions were recommended to the patients. In subsequent iteration, we plan to provide alerts to the patients that can lead to patient (or his/her guardian) actions subject to advanced instruction provided by the doctor, or encourage the patient to contact the doctor when advance instruction does not specify an action that the patient (or his/her guardian) can take on his/her own. 


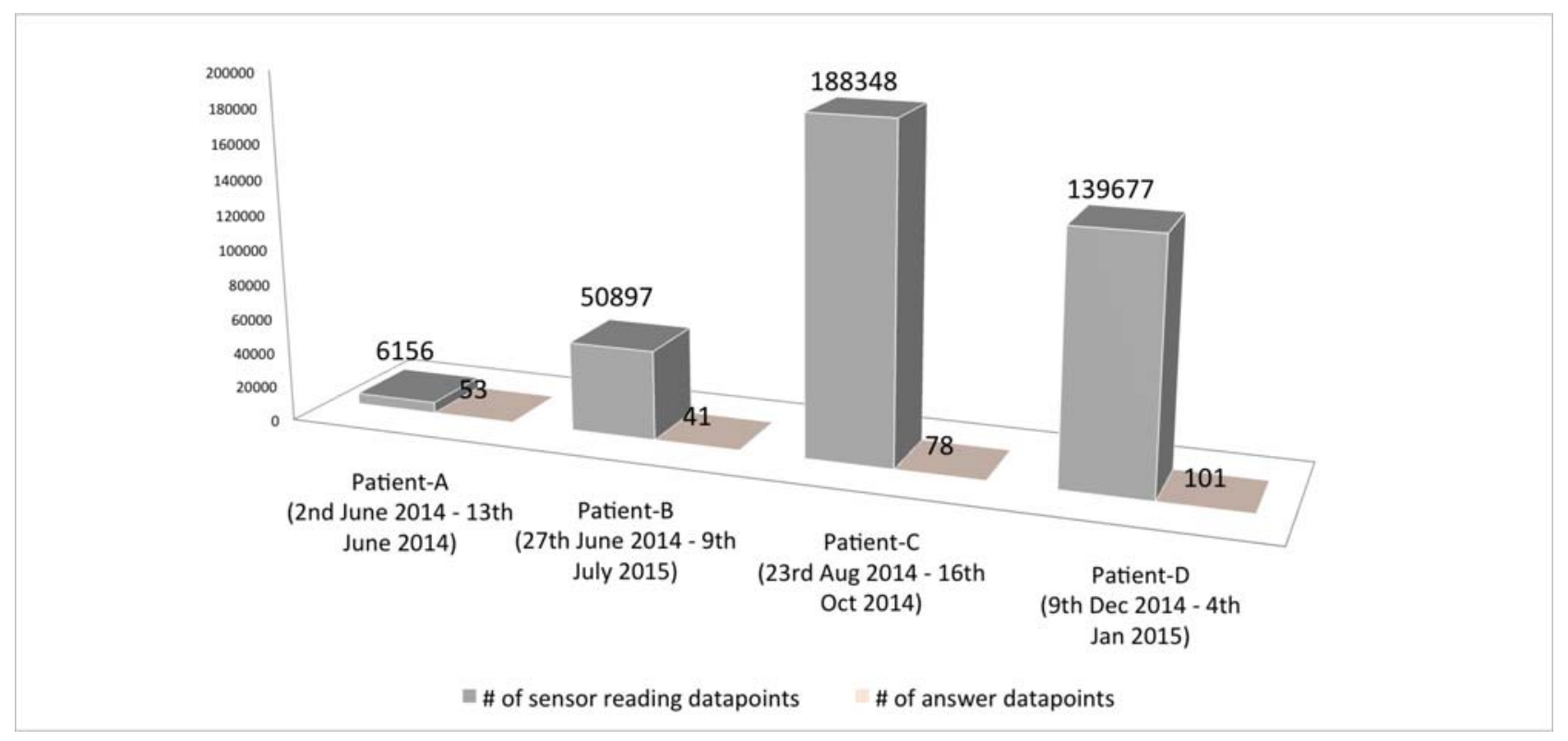

Fig. 4. Number of data points gathered from four patients with asthma; sensor data and answers to questionnaire reported separately for Patient-A, Patient-B, Patient-C, and Patient-D

\section{KHEALTH Kit DEPLOYMENT StUdy}

After iterative development and testing of the kHealth application by doctors and developers, the kit was deployed for use with multiple patients in a real-world setting under the IRB (The Dayton Childrens Hospital (DCH) Study IRB 2013-035, October 2013; addendum 04/02/2014; with informed consent, demographic survey, initial survey, final survey, asthma tracking form). We outline the deployment process, data collection, and preliminary data analysis resulting in some promising insights.

\section{A. Deployment and Data Collection}

kHealth for asthma application has gone through multiple iterations of deployment and development and has evolved over time since its inception. Subjects were recruited by advertising for test subjects in the doctor's clinic. kHealth kits for asthma were given to four patients and it is now being used with an additional sensor to monitor sleep quality of asthma patients. Out of four patients, three patients had a good control over asthma during our study. One patient had asthma symptom and had activity limitation and cough during our study. We call this patient Patient-A. For clarity and interestingness, we focus on the data from one patients who exhibited some symptoms of asthma that resulted in interesting insights. Figure 4 summarizes the data points we collected during a deployment from June 2nd to June 13th, 2014 for Patient-A.

\section{B. Preliminary Data Analysis}

We analyzed the observations from sensors (numerical) and answers to questionnaire (yes/no) in a holistic setting guided by domain expert's knowledge. We could find four important insights (referred to as hypothesis in the rest of the paper) from our preliminary data analysis of Patient-A discussed in detail here:
H1: Activity limitation related to high exhaled Nitric Oxide: Figure 5(a) shows a plot of exhaled nitric-oxide and the answers to a question that Patient-A has provided from June 2nd to June 13th, 2014. When the patient reported limitation in activity, the exhaled nitric-oxide is observed to be high. When the patient reported no limitation on activity, a decreasing trend in the exhaled nitric-oxide was observed. On June 8th patient reported no activity limitation and the corresponding date has minimum exhaled nitric-oxide.

H2: Activity limitation observed with high pollen activity: Figure 5(b) has a plot of external pollen level and answer to a question related to the activity limitation of a patient. Patient-A's activity limitation is observed with the high pollen count in the environment. On June 8th patient reported no activity limitation and the corresponding date has minimum pollen count.

H3: Low exhaled Nitric Oxide observed with absence of coughing: Figure 5(c) has a plot of exhaled nitric-oxide and the answers to a question on coughing and chest tightness. Patient-A's coughing and chest tightness happen to be on days when the exhaled nitric-oxide was higher. On June 8th patient reported no symptom of cough or chest tightness and the corresponding day has the minimum exhaled nitric-oxide.

H4: Medication (Albuterol) related to decreasing Exhaled Nitric Oxide: Figure 5(d) contains a plot of exhaled nitricoxide and the answers to a question on taking albuterol (medication). Patient-A's answer of "yes" to the medication appears with the decreasing trend of exhaled nitric-oxide. This observation is on June 8th when the patient indicated that the medication was taken.

\section{DISCUSSION}

The insights we presented $(\mathrm{H} 1, \mathrm{H} 2, \mathrm{H} 3$, and $\mathrm{H} 4)$ are still in the preliminary stage and has been validated to be interesting by the doctor. However, a large scale evaluation is 
(a)

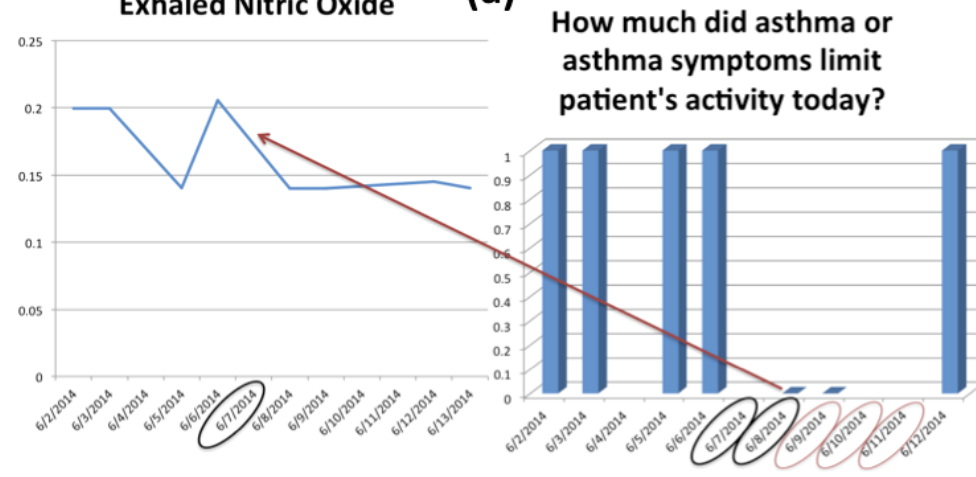

(c)

Exhaled Nitric Oxide

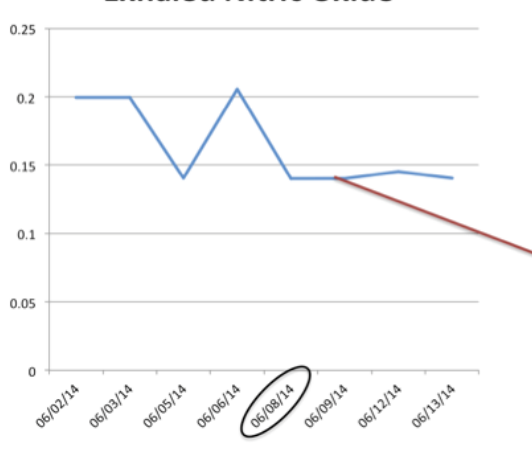

Has patient had wheeze, chest tightness, or asthma related cough today?

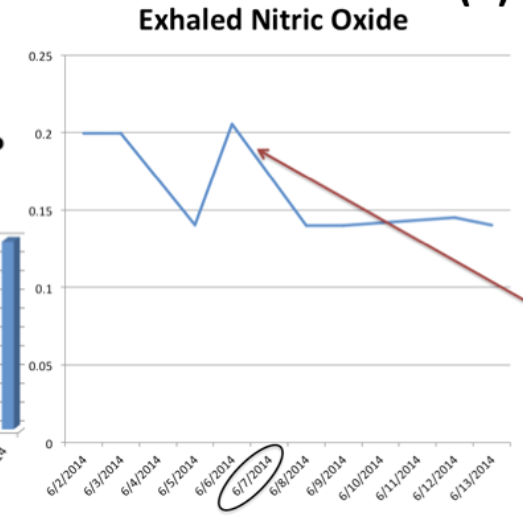

How much did asthma or asthma symptoms limit patient's activity today? (b)

(d)

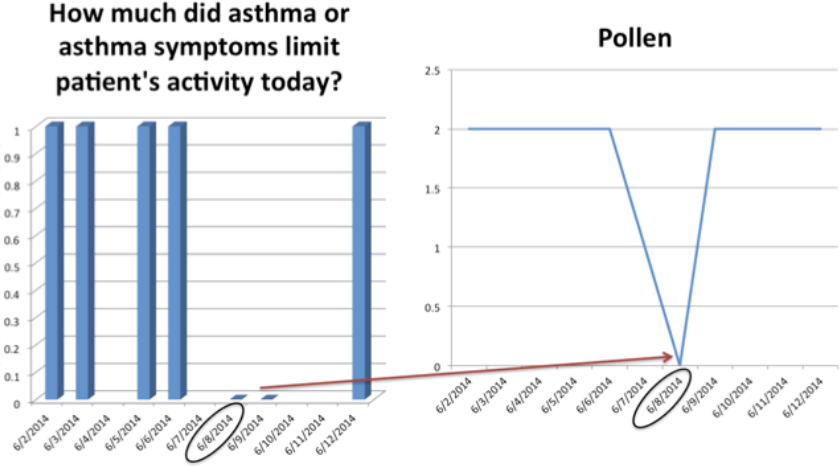

Fig. 5. Insights gleaned from the preliminary data collection from the kHealth kit deployment. The Nitric Oxide readings are in parts per million (ppm), pollen readings are in units, answers to questions are on a scale of 0 to 3: 0 being minor effect to 3 being major effect

required for gathering evidence for the derived hypothesis. We summarize some of our experiences in designing, developing, and deploying the kHealth kit by taking an iterative approach.

\section{A. Disambiguation by Adding New Sensors}

There are two orthogonal insights we gained in terms of adding new sensors for monitoring asthma patients: (1) It is beneficial to add sensors to disambiguate/distinguish between multiple causes. For example, in the second hypothesis (H2), we observe decreasing exhaled nitric-oxide and decreasing pollen count in the patient's environment. For establishing this connection, we need to gather corroborative evidence. For example, if the patient indicates that she is sensitive to a particular type of pollen and we observe that in the environment (assuming we have a sensor to do so), we can infer a stronger association in $\mathrm{H} 2$. $\mathrm{H} 2$ utilizes both person level and public level signals for a better understanding of the individual's symptoms. (2) It is beneficial to add sensors to capture some of the Activities of Daily Living (ADL) e.g., disturbed sleep can be inferred using a Fitbit ChargeHR [51]. A continuous capture and analysis of some of the physiological parameters of the patient can also help provide a more in-depth understanding of patient's asthma symptoms.

\section{B. Challenges in Data Management}

Passive sensor platform like sensordrone [19] has over ten sensors collecting environmental observations. The sampling rate of each of these sensors is around one second leading to 86,400 data points for each sensor. A total of 864,000 data points $(86,400$ data points/sensor $\times 10$ on-board sensors $)$ are generated by all the on-board sensors on sensordrone. Passive sensing extends to monitoring external environment such as outdoor humidity, temperature, pollen, and air quality leading to a massive amount of data. We faced challenges in managing data collected from all the sensors on the mobile platform. We devised a strategy for storing only when there is a change in value for a passive sensor observation. For example, say, $\mathrm{x}$ is the variable assigned an initial value $a_{1}$ of carbon-monoxide reading at time $t_{1}$. We push the value to a SQLite database on the mobile device at time $t_{1}$. At next time step $t_{2}$, we push the value to the database only if the value of carbon-monoxide reading at $t_{2}$ is not equal to $a_{1}$. We store carbon-monoxide readings only when there is a change in the reading. Effective strategies for decision on storing observations and techniques of semantic abstractions [52], [9] will greatly help in dealing with the volume of data. Out strategy of storing observations only upon change from its previous value reflects in the number of sensor data points being stored for each patient in Figure 4.

\section{Challenges in User Training}

Active sensing part of the kHealth kit requires involvement of users e.g., answering the questionnaire. External sensors such as sensordrone and NODE sensor platforms requires patients to be aware of basic Bluetooth connection procedures such as paring/un-pairing of devices. If the sensors lose charge, 
they have to be re-paired before the observations can be acquired from them.

All the devices used in the kHealth kit needs periodic charging. The duration of charging and the battery life depends on the usage and varies significantly across devices. Users found this challenging, especially due to multiple devices in the kit. Providing reminders to charge the devices would be a workaround. A single platform like a mobile phone with embedded sensors for asthma management would completely remove the challenge of handling multiple devices.

Moving toward passive sensing will minimize user involvement, reducing effort and training required to use the kHealth kit. We are in the early stages of creating such as passive sensing system which may act as proverbial canary in a coal mine. Right now, active sensing component of kHealth kit involves answering questions on a mobile device and collect exhaled nitric-oxide. External sensors add complexity in terms of usage, communication, and data storage. So, we have created an extensive documentation on using the mobile application with the external sensors which can be found on the project page [53].

\section{CONCLUSION AND FUTURE WORK}

Asthma management in children is a crucial problem. kHealth kit for asthma is capable of collecting and summarizing data from both active and passive sensors, utilizing the computational power of mobile devices and low-cost sensors. Real-world deployment of the kit exposed some of the challenges in data collection, usability, and communication. The preliminary analysis of the data collected from patients resulted in promising insights. Specifically, we observed the correlation between patient's exhaled nitric-oxide and pollen level on activity limitation, coughing, and effect of taking medication.

As a future work, we plan to add more passive sensors for monitoring activity, sleep, breathing rate etc., that will help us gain further insights into possible triggers and their impact on asthma patients. Such an understanding would lead to proactive, personalized, and actionable information for preventing asthma attacks and to improve the quality of life.

\section{ACKNOWLEDGMENT}

We thank Dan Vanuch for contribution to the development the Android application, creating user manual, and tutorial for using the kHealth kit for asthma. Partial support for this research was provided by Wright State University's VP of Research under a challenge grant.

\section{REFERENCES}

[1] (2015) NHLBI description of asthma. [Online]. Available: http: //www.nhlbi.nih.gov/health/health-topics/topics/asthma/

[2] (2015) Asthma related facts. [Online]. Available: http://www.lung.org/ lung-disease/asthma/resources/facts-and-figures/asthma-in-adults.html

[3] D. A. Squires. (June 2011) The u.s. health system in perspective: A comparison of twelve industrialized nations. [Online]. Available: http://bit.ly/oZwhFZ

[4] (2015) Health costs: How the u.s. compares with other countries. [Online]. Available: http://www.pbs.org/newshour/rundown/2012/10/ health-costs-how-the-us-compares-with-other-countries.html
[5] L. J. Akinbami, J. E. Moorman, P. L. Garbe, and E. J. Sondik, "Status of childhood asthma in the united states, 1980-2007," Pediatrics, vol. 123, no. Supplement 3, pp. S131-S145, 2009.

[6] (2015) Quantified self. [Online]. Available: http://quantifiedself.com/

[7] (2015) khealth: A knowledge-enabled semantic platform to enhance decision making and improve health, fitness, and well-being. [Online]. Available: http://knoesis.org/projects/khealth

[8] P. J. Hu, P. Y. Chau, O. R. L. Sheng, and K. Y. Tam, "Examining the technology acceptance model using physician acceptance of telemedicine technology," Journal of management information systems, pp. 91-112, 1999.

[9] C. A. Henson, "A semantics-based approach to machine perception," $\mathrm{Ph}$.D. dissertation, Wright State University, 2013.

[10] M. C. Shults, R. K. Rhodes, S. J. Updike, B. J. Gilligan, and W. N. Reining, "A telemetry-instrumentation system for monitoring multiple subcutaneously implanted glucose sensors," Biomedical Engineering, IEEE Transactions on, vol. 41, no. 10, pp. 937-942, 1994.

[11] S. Vaddiraju, I. Tomazos, D. J. Burgess, F. C. Jain, and F. Papadimitrakopoulos, "Emerging synergy between nanotechnology and implantable biosensors: a review," Biosensors and Bioelectronics, vol. 25, no. 7, pp. 1553-1565, 2010.

[12] J. S. Karlsson, U. Wiklund, L. Berglin, M. Östlund, M. Karlsson, T. Bäcklund, K. Lindecrantz, and L. Sandsjö, "Wireless monitoring of heart rate and electromyographic signals using a smart t-shirt," in Proceedings of International Workshop on Wearable Micro and Nanosystems for Personalised Health. Citeseer, 2008.

[13] S. Ishida, N. Shiozawa, Y. Fujiwara, and M. Makikawa, "Electrocardiogram measurement during sleep with wearing clothes using capacitively-coupled electrodes," in Engineering in Medicine and Biology Society, 2007. EMBS 2007. 29th Annual International Conference of the IEEE. IEEE, 2007, pp. 2647-2650.

[14] (2015) The wyss institute at harvard university. [Online]. Available: http://www.bu.edu/abl/files/footwearinsight.pdf

[15] M. Chan, E. Campo, D. Estève, and J.-Y. Fourniols, "Smart homescurrent features and future perspectives," Maturitas, vol. 64, no. 2, pp. 90-97, 2009

[16] V. Rialle, F. Duchene, N. Noury, L. Bajolle, and J. Demongeot, "Health" smart" home: information technology for patients at home," Telemedicine Journal and E-Health, vol. 8, no. 4, pp. 395-409, 2002.

[17] M. Uenoyama, T. Matsui, K. Yamada, S. Suzuki, B. Takase, S. Suzuki, M. Ishihara, and M. Kawakami, "Non-contact respiratory monitoring system using a ceiling-attached microwave antenna," Medical and Biological Engineering and Computing, vol. 44, no. 9, pp. 835-840, 2006.

[18] Y. G. Lim, K. K. Kim, and K. S. Park, "ECG recording on a bed during sleep without direct skin-contact," Biomedical Engineering, IEEE Transactions on, vol. 54, no. 4, pp. 718-725, 2007.

[19] (2015) Sensordrone, a bluetooth enabled lowcost sensor for monitoring the environment. [Online]. Available: http://www.kickstarter.com/projects/453951341/ sensordrone-the-6th-sense-of-your-smartphoneand-be/

[20] E. J. Topol, The creative destruction of medicine: How the digital revolution will create better health care. Basic Books, 2012.

[21] A. Wood, J. A. Stankovic, G. Virone, L. Selavo, Z. He, Q. Cao, T. Doan, Y. Wu, L. Fang, and R. Stoleru, "Context-aware wireless sensor networks for assisted living and residential monitoring," Network, IEEE, vol. 22, no. 4, pp. 26-33, 2008.

[22] Z. Lv, F. Xia, G. Wu, L. Yao, and Z. Chen, "icare: a mobile health monitoring system for the elderly," in Proceedings of the 2010 IEEE/ACM Int'l Conference on Green Computing and Communications \& Int'l Conference on Cyber, Physical and Social Computing. IEEE Computer Society, 2010, pp. 699-705.

[23] T. Banerjee, M. Rantz, M. Li, M. Popescu, E. Stone, M. Skubic, and S. Scott, "Monitoring hospital rooms for safety using depth images," AI for Gerontechnology, Arlington, Virginia, US, 2012.

[24] T. Banerjee, J. Keller, M. Skubic, and E. Stone, "Day or night activity recognition from video using fuzzy clustering techniques," 2013.

[25] T. Banerjee, M. Skubic, J. M. Keller, and C. Abbott, "Sit-to-stand measurement for in-home monitoring using voxel analysis," Biomedical 
and Health Informatics, IEEE Journal of, vol. 18, no. 4, pp. 1502-1509, 2014.

[26] E. E. Stone and M. Skubic, "Evaluation of an inexpensive depth camera for passive in-home fall risk assessment," in Pervasive Computing Technologies for Healthcare (PervasiveHealth), 2011 5th International Conference on. IEEE, 2011, pp. 71-77.

[27] H. Nait-Charif and S. J. McKenna, "Activity summarisation and fall detection in a supportive home environment," in Pattern Recognition, 2004. ICPR 2004. Proceedings of the 17th International Conference on, vol. 4. IEEE, 2004, pp. 323-326.

[28] (2015) FDA approved first digital pill. [Online]. Available: http://www.forbes.com/sites/singularity/2012/08/09/ no-more-skipping-your-medicine-fda-approves-first-digital-pill/

[29] (2015) Qualcomm tricorder $x$ prize. [Online]. Available: http: //www.qualcommtricorderxprize.org/

[30] (2015) Nokia sensing x challenge. [Online]. Available: http://www. nokiasensingxchallenge.org/

[31] (2015) The usc center for body computing. [Online]. Available: http://www.uscbodycomputing.org/

[32] (2015) Harvard sensor network lab. [Online]. Available: http: //fiji.eecs.harvard.edu/CodeBlue

[33] (2015) Kickstarter, bringing creativity to life. [Online]. Available: http://www.kickstarter.com/

[34] (2015) e-health sensor platform for raspberry pi and arduino. [Online]. Available: http://www.kickstarter.com/

[35] Y. Qiu, A. Whittaker, M. Lucas, and K. Anderson, "Automatic wheeze detection based on auditory modelling," Proceedings of the Institution of Mechanical Engineers, Part H: Journal of Engineering in Medicine, vol. 219, no. 3, pp. 219-227, 2005.

[36] M. Wisniewski and T. Zielinski, "Digital analysis methods of wheezes in asthma," in Signals and Electronic Systems (ICSES), 2010 International Conference on. IEEE, 2010, pp. 69-72.

[37] Z. Moussavi, "Fundamentals of respiratory sounds and analysis," Synthesis lectures on biomedical engineering, vol. 1, no. 1, pp. 1-68, 2006.

[38] J. Zhang, W. Ser, J. Yu, and T. Zhang, "A novel wheeze detection method for wearable monitoring systems," in Intelligent Ubiquitous Computing and Education, 2009 International Symposium on. IEEE, 2009, pp. 331-334.

[39] (2015) Mobile line]. Available: asthma monitoring. [Onqualcomm-life-isonea-to-offer-mobile-asthma-monitor/

[40] (2015) Acoustic respiratory monitoring technology by isonea. [Online]. Available: http://www.isoneamed.com/

[41] D. Oletic, "Wireless sensor networks in monitoring of asthma," 2009.

[42] H. Gong, "Clinical methods: The history, physical, and laboratory examinations," 1990

[43] E. Y. Seto, A. Giani, V. Shia, C. Wang, P. Yan, A. Y. Yang, M. Jerrett and R. Bajcsy, "A wireless body sensor network for the prevention and management of asthma," in Industrial Embedded Systems, 2009. SIES'09. IEEE International Symposium on. IEEE, 2009, pp. 120 123.

[44] I.-J. Chen and H.-W. Chiu, "A review for information systems to support outpatient asthma health management," 2009.

[45] (2015) Propeller health (formerly, asthmapolis) website. [Online]. Available: http://propellerhealth.com/

[46] E. C. Vasbinder, H. M. Janssens, M. P. Rutten-van Mölken, L. van Dijk, B. C. de Winter, R. C. de Groot, A. G. Vulto, P. M. van den Bemt et al., "e-monitoring of asthma therapy to improve compliance in children using a real-time medication monitoring system ( $\mathrm{rtmm})$ : the ematic study protocol," BMC medical informatics and decision making, vol. 13 , no. 1 , p. $38,2013$.

[47] (2015) Asthma related research at microsoft research cambridge. [Online]. Available: http://www.microsoft.com/en-us/researchconnections/ science/stories/machine-learning.aspx

[48] (2015) Manchester asthma study. [Online]. Available: http://www. maas.org.uk/default.html

[49] (2015) Expert panel report 3 (epr3): Guidelines for the diagnosis and management of asthma. [Online]. Available: http://www.nhlbi.nih.gov/ guidelines/asthma/asthgdln.htm

[50] T. Banerjee, P. Anantharam, W. L. Romine, L. Lawhorne, and A. Sheth, "Evaluating a potential commercial tool for healthcare application for people with dementia," 2015 (accepted).

[51] (2015) Fitbit chargehr. [Online]. Available: https://www.fitbit.com/ chargehr

[52] C. Henson, A. Sheth, and K. Thirunarayan, "Semantic perception: Converting sensory observations to abstractions," Internet Computing, IEEE, vol. 16, no. 2, pp. 26-34, 2012.

[53] (2015) Asthma project page and resources. [Online]. Available: http://wiki.knoesis.org/index.php/Asthma 